\title{
Sensitivity study on forest fire breakout and propagation conditions for forest fire hazard curve evaluations
}

\author{
Yasushi OKANO* and Hidemasa YAMANO* \\ *Japan Atomic Energy Agency \\ 4002 Narita, Oarai, Ibaraki 311-1393, Japan \\ E-mail: okano.yasushi@jaea.go.jp
}

Received: 15 September 2016; Accepted: 19 February 2017

\begin{abstract}
As a part of external hazard probabilistic risk assessment methodology development, a sensitivity study on forest fire hazard curves was performed on condition parameters where frequency/probability variables were varied within respective fluctuation ranges. Important variables related to forest fire breakout time, forest fire breakout points, and forest firefighting operation were identified. The probability fluctuation on forest fire breakout time only affects the intensities of the hazard curves, but not the frequency, because the intensity increases in daytime due to sunshine and the breakout probability in daytime is statistically higher than the daily average. The hazard curves of the reaction intensity and the fireline intensity increased around $4 \%$ and $14 \%$ respectively in comparison with those without the forest fire breakout time fluctuation. The probability fluctuation on forest fire breakout points affects only the frequency of the hazard curves, but not the intensities. The hazard curves vary around $+70 \%$ to $-40 \%$ in frequency, because each forest fire breakout point has different distance to the plant and the forest fire arrival probability varies with propagation duration. The probability fluctuation due to forest firefighting operation only affects the frequency of the hazard curves, but not the intensity. The effect of the forest firefighting operation was conservatively assumed where there is no forest firefighting operation outside a plant, hence all potential forest fires arrive at the plant. The hazard curves remarkably increase around 40 to 80 times in frequency in comparison with those with considering the forest firefighting operation effect outside the plant. This study indicated that the most significant factor in the forest fire hazard risk is whether the forest firefighting operation outside the plant is expected before the forest fire arrival at the plant.
\end{abstract}

Key words: Forest fire, External hazard, Probabilistic risk assessment, Hazard curve, Reaction intensity, Fireline intensity, Sensitivity study, Sodium cooled fast reactor

\section{Introduction}

Probabilistic risk assessment (PRA) is utilized not only to quantify a core damage frequency of a nuclear power plant (NPP) but also to analyze weak points of an NPP to enhance the safety. Continuous efforts are necessary to develop a PRA method and to apply it to future NPP system designs. Safety concerns over various external hazards on NPPs were recognized especially after the Fukushima Daiichi NPP accidents. A new regulation established in Japan after the accidents has defined a forest fire as one of external hazards to be assessed (Nuclear Regulation Authority (NRA), 2013). Safety assessments on a forest fire have been performed with a deterministic approach for the time being, in which forest fire propagation simulations are performed under conservative initial and boundary conditions. A forest fire breakout near an NPP is postulated to assess the integrity of NPP systems against forest fire effects, e.g. a reactor building against radiation heat, a diesel generator system against smoke. In the assessment, forest fire breakout locations are selected considering potential forest fire causes and land utilizations near the NPP, then reaction intensity and fireline intensity around the NPP are evaluated with the worst weather overlapping condition by a numerical simulation of forest fire propagation using e.g. Fire Area Simulator (FARSITE) (Finney, 2004). Here, the "reaction intensity" (unit in $\mathrm{kW} / \mathrm{m}^{2}$ ) represents a heat release rate per unit area by a combustion of forest fuel (e.g. trees and 
leaves, fallen leaves, and underbrush), which mainly depends on vegetation and moisture content in the forest fuel. The "fireline intensity" (unit in $\mathrm{kW} / \mathrm{m}$ ) represents a torrential movement of frontal fire by a combination of reaction intensity and fire propagation speed, which depends on reaction intensity, wind speed, and topography. However, this deterministic approach is not enough to make a quantitative comparison of relative risk of a forest fire to other external hazard risks. A risk assessment based on a PRA is necessary; i.e. a hazard curve where a forest fire with certain intensity and frequency happens as well as a fragility curve with conditional probability of a failure of a structure, system and component of an NPP caused by a forest fire are necessary to be obtained.

Past studies of a risk assessment on a forest fire (Song, et al., 2006, Sano, 2005, Braun et al., 2010, and Preisler et al., 2004) focused on the frequency of occurrence based on a statistical approach using forest fire databases, and frequency-intensity combination has not been assessed. These past studies were mainly for "hazard map" evaluations, and scope ranges of areas were a prefecture, a state, and/or a country, and the map resolutions were around $\mathrm{km} \times \mathrm{km}$ or larger mesh sizes. In order to evaluate the effects on an NPP, higher resolution evaluation near the NPP, including local topographical and land utilization features (e.g. brooks, valleys, and ridges of hills and mountains), is indispensable.

Our previous study (Okano and Yamano, 2015a) provided a new method to evaluate a hazard curve of a forest fire where a combination of frequency and intensity is obtained based on fine resolution evaluations. There are physical phenomena caused by forest fire, and the assessments have been performed for the effects by heat and flame (Okano and Yamano, 2015b), smoke (Okano and Yamano, 2016), and flying sparks. In order to obtain the hazard curve on the heat effect, high-resolution FARSITE simulations (10 $\mathrm{m} \times 10 \mathrm{~m}$ meshes in several $\mathrm{km} \times \mathrm{km}$ area around an NPP) were performed for hundreds of cases with various weather conditions such as prevailing wind speed (PWS) and relative humidity $(\mathrm{RH})$ and with several forest fire breakout locations to evaluate response surfaces. The response surfaces are utilized to provide the reaction intensity and the fireline intensity in a certain Monte Carlo sample which includes a set of condition variables related to "forest fire breakout and propagation" and "weather". The response surfaces are approximated by the Gaussian process interpolation (Okano and Yamano, 2015a) whose accuracy (i.e. the root mean squared error divided by the average value) is $\pm 32 / 863=0.037$ for the reaction intensity and $\pm 17 / 227=0.075$ for the fireline intensity. Typical hazard curves of the reaction intensity and the fireline intensity are shown in the upper half of Fig. 1, which present the annual exceedance frequency of the intensities.

\section{Image of typical forest fire hazard curves}
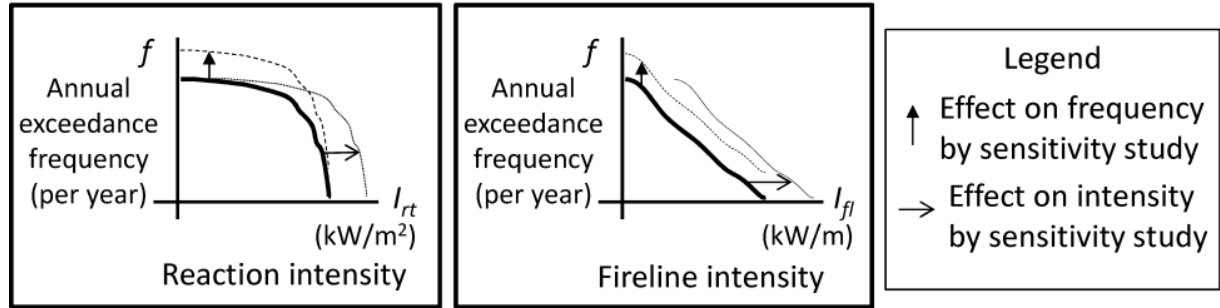

\section{Selected logic boxes of hazard curve evaluation for sensitivity study}

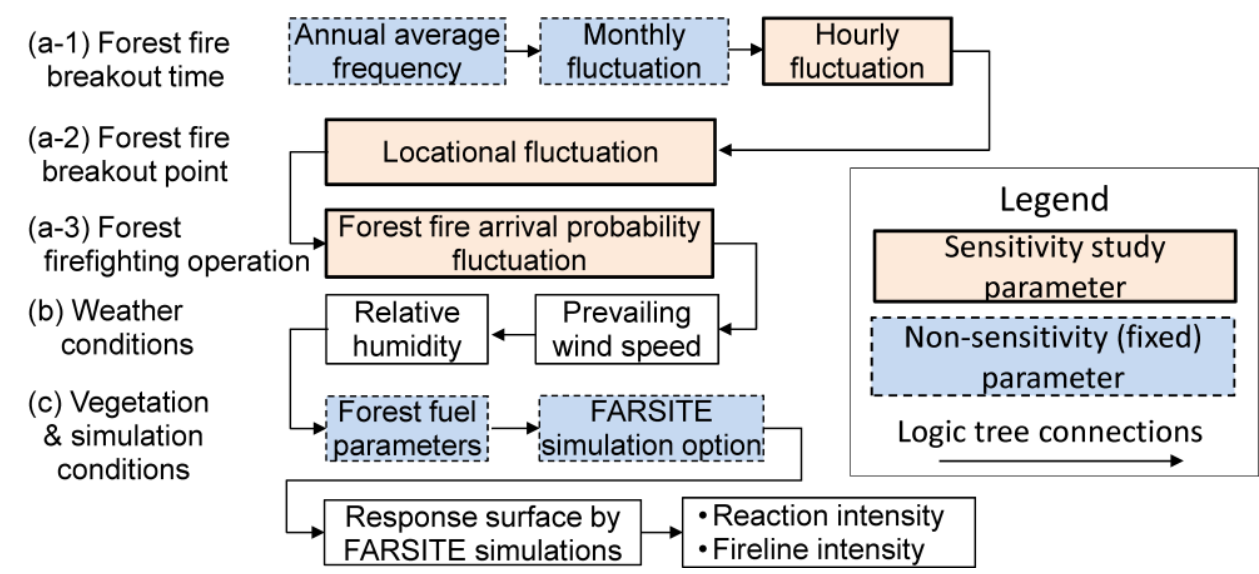

Fig. 1 Typical changes of forest fire hazard curves are illustrated on the top. Logic boxes affecting forest fire intensity are illustrated on the bottom. Variables of light orange logic boxes were selected for the sensitivity study. Parameters of light blue logic boxes do not change during a forest fire and are fixed. Appearance probabilities of weather condition in white logic boxes are utilized in a Monte Carlo simulation for the hazard curve evaluations. 
This paper specifically focuses on a sensitivity study on hazard curves of reaction intensity and fireline intensity due to fluctuations of condition parameters related to forest fire breakout and propagation. Hazard curves of the forest fire intensity are quantitatively compared with those of typical regional conditions around NPPs including a sodium-cooled fast reactor which had been obtained in our previous study. A forest fire record (Crisis Measures and Disaster Prevention Division, 2013), meteorological data (Japan Meteorological Agency, 2013) and vegetation data (Ministry of Land, Infrastructure, Transport and Tourism (MLIT), 2013, and Forestry Division, 2013) of the studied area, which is near a typical sodium-cooled fast reactor in Japan, are referred to the evaluations.

\section{General consideration on physical effects of logic box parameters on forest fire hazard curves}

The hazard curve evaluation method developed in our previous study utilizes a logic tree (Okano and Yamano, 2015a). The logic tree consists of categorized domains: "forest fire breakout and propagation" condition, "weather" condition, "vegetation" condition, and "forest fire simulation" condition. Each domain contains logic boxes which represent particular conditions having influences on the intensity, and each logic box has a static or probabilistic/frequency parameter according to the parameter state during a forest fire. A constant parameter is given for the one unchanged during forest fire duration (typically several hours to days) or having low sensitivity to the reaction intensity and the fireline intensity. A probability or frequency is given to a variable (i.e. non-static parameter) based on a relevant statistical database or a numerical evaluation using a physical model.

For the sensitivity study on the hazard curves, logic boxes were analyzed at first from the viewpoints of general characteristics which change the frequency and/or intensity of forest fire hazard curves. Key points are that the hazard curve is derived as an "annual" exceedance frequency based on the annual average and areal average conditions so that the condition parameters of the logic boxes with significant fluctuations or deviations are focused on as the sensitivity study. The logic boxes selected for this sensitivity study are summarized in the lower half of Fig. 1.

Our previous study on a forest fire propagation simulation around an NPP in Japan indicated that the forest fire intensity has sensitivity to PWS and RH among other weather condition parameters (Okano and Yamano, 2015b). According to forest fuel moisture models (Rothermel et al., 1986), sunshine on a forest increases forest fuel temperature higher than ambient air, leading to decrease in $\mathrm{RH}$ in the forest. As a result, the reaction intensity and the fireline intensity increase. The sun shines only in daytime. Furthermore, a forest fire statistically tends to break out in the daytime more than in the nighttime because the main causes of forest fire are related to human activities that increase in the daytime in general. Hence, daytime-biased (i.e. higher) fluctuation of forest fire breakout frequency will affect not only the momentary frequency but also the intensity of the hazard curve. Therefore, a fluctuation related to forest fire breakout time is selected for the sensitivity study.

No hazard map showing frequent points of forest fires in the studied area has been developed so far. However there are potential breakout points around the NPP deriving from human activities and accessibility: e.g. an end of forest road, public beaches, and campsites. Each point differs from one another in areal and temporal densities of human activities and in distance from the NPP. Probability distribution among the selected potential breakout points fluctuates depending on human activities. For example, controlled burn is usually done in spring in Japan, cooking out on beaches or in campsites is popular in spring and summer, and public beaches are open throughout a year. The forest fire breakout probability distribution (i.e. breakout frequency of each breakout point) will fluctuate during a year, and a fluctuation related to forest fire breakout points is selected for the sensitivity study.

Forest firefighting operation extinguishes a forest fire or terminates forest fire propagation, and it affects the probability of forest fire arrival at an NPP. Statistically, forest firefighting operations suppress most forest fires within one to two hours in Japan. The effectiveness of firefighting depends on accessibility for fire engines to a forest fire area and on positional relation to the fire area, access routes, and leeward/windward wind direction. In general, an NPP is located far from city urban area in Japan, accordingly with sparse road network. The forest firefighting operation is usually performed only in daytime. It is reported that, after a disaster (e.g. a severe earthquake), it takes several times longer for fire engines to arrive at a fire site than usual because of access road damage and/or fire station damages thus firefighting operations tend to be delayed. Therefore, the probability of forest fire arrival at an NPP will fluctuate due to the existence and the effectiveness of the forest firefighting operation around the NPP, and a fluctuation related to forest firefighting operation is selected for the sensitivity study.

Based on above, three items for the sensitivity study are selected as shown in the lower half of Fig. 1 in which the 
selected logic boxes are depicted:

$\checkmark \quad$ "Hourly fluctuation" box in Fig. 1 is representative of the forest fire breakout time,

$\checkmark \quad$ "Locational fluctuation" box in Fig. 1 is representative of the forest fire breakout point, and

$\checkmark \quad$ "Forest fire arrival probability fluctuation" box in Fig. 1 is representative of the forest firefighting operation.

\section{Identification of parameter ranges of sensitivity study 3.1 Range of hourly fluctuation of forest fire breakout time}

The annual average forest fire breakout frequency in the studied area is 0.36 per year, and the momentary frequency fluctuates throughout a year. The left figure of Fig. 2 compares the fluctuation of the prefecture of the studied area and that of all over Japan. The data is from the prefecture forest fire database (Crisis Measures and Disaster Prevention Division, 2013) and from the reference for all over Japan (Forest Fire Control Association, 2007). As for the fluctuation within a day, probability in the late morning and in the afternoon (the peak is during 10 to 18 o'clock) is higher than a daily average in both the prefecture and all over Japan cases, which corresponds to the main cause of forest fires by human activities. For example, there were 67 forest fires in the prefecture between 2004 and 2012; and 52 cases were related to human activities, one case was by lightening, and 14 cases were by other or unknown causes. In the studied area, the average of the peak hour is 2.8 times higher than the daily average as shown in the right figure of Fig. 2. On the other hand, the nighttime average is around 1/9 (=0.11) of the daily average. As for the monthly fluctuation, a frequency of forest fire breakout in April is around 4 times higher than the annual average in the area. The hazard curve evaluation includes these fluctuations of the frequency when summarizing "annual" exceedance frequency, although the daytime sunshine effects on the intensity that will change the hazard curve should also be considered in the evaluation. In this study, the forest fire breakout probability fluctuation, which increases 2.8 times in daytime peak hour (10-18 o'clock) and decreases around 1/9 in nighttime (18-10 o'clock), is utilized, whereas the monthly fluctuation is not counted due to that changes in sunshine effect between summer and winter are smaller than that between daytime and nighttime (i.e. with or without sunshine), and the monthly peak timing, i.e. April, is near the spring equinox and the sunshine intensity is near the yearly average. The hazard curves are evaluated in this study that the probability distribution in a day (i.e. daytime peak hour and nighttime) in order to compare to the reference case where the probability is equal through a day.
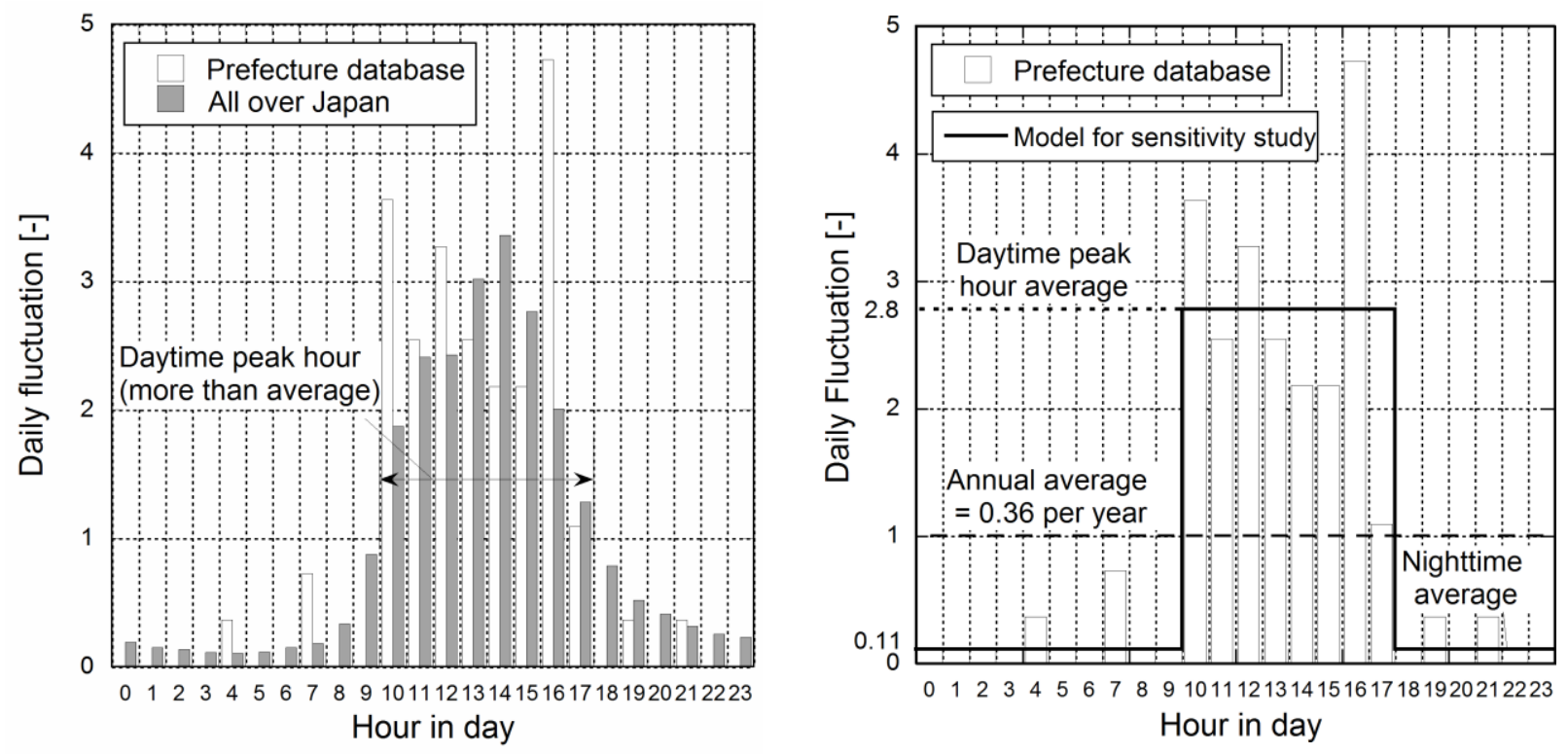

Fig. 2 Fluctuations of probability of breakout time during a day of the prefecture which includes the studied area and all over Japan are shown in the left figure. The daily fluctuation model for the sensitivity study which has daytime peak duration (i.e. 10-18 o'clock) and nighttime duration (i.e. 18-10 o'clock) is shown in the right figure. 


\subsection{Range of locational fluctuation of forest fire breakout points}

Three forest fire breakout points were selected considering land utilization and areas of human activities: one forest road end $2.5 \mathrm{~km}$ south-south east from the NPP, and two public beaches $3.5 \mathrm{~km}$ east and $1 \mathrm{~km}$ south-west from the NPP. The hazard curves are evaluated in this study that the probability distribution (i.e. weighting factor) varied among three points where one point has higher probability than the other two points in order to compare to the reference case where the probability is equal through three points. In the studied area, principal forest species are deciduous broad-leaf trees, and some areas are composed of evergreen needle-leaf trees or evergreen cedar trees. According to the past study related to forest species and forest fire occurrences in Japan (Satoh, 2005), the breakout rate (i.e. the number of fired areas per one year out of total investigated areas which had been statistically investigated for all over Japan) of deciduous broad-leaf trees, evergreen needle-leaf trees, and evergreen cedar trees are $0.37 \%$ per year, $0.13 \%$ per year and $0.61 \%$ per year, respectively. The ratio of the forest fire of the most dominant species to the total three species are around 0.6 (i.e. $0.61 /(0.37+0.13+0.61)=0.55$, then rounded off to 0.6$)$. It is assumed for the sensitivity study that the most frequent breakout point has the probability of 0.6 and the other two points share the rest probability (i.e. (1-0.6) / $2=0.2$ for each point).

\subsection{Range of forest fire arrival probability fluctuation by forest firefighting operation}

The probability of forest fire arrival at an NPP is a function of a grace period (i.e. duration of forest fire propagation from its breakout point to the NPP) because forest firefighting operations are expected during the grace period. In our previous study, the probability of the arrival at an NPP $\left(p_{\text {arv }}\right)$ was formulated using a complementary probability of extinguishment of a forest fire during the grace period. The Weibull distribution was applied to model the probability (Okano and Yamano, 2015a) as:

$$
p_{\text {arv }}(t)=\exp \left\{-[(t-\gamma) / \eta]^{m}\right\}
$$

where $t$ means the arrival time after the breakout, and $\gamma(\equiv 1), \eta(=0.47)$, and $m(=0.48)$ are location, scale, and shape parameters of the Weibull distribution, respectively. Conversely, for a pessimistic case that the forest firefighting cannot be expected at all, the forest fire is assumed not to be extinguished and the probability becomes unity as:

$$
p_{\text {arv }}(t)=1
$$

The hazard curve without accounting the forest firefighting effect (i.e. using the Eq. (2)) is a conservative assumption because not only some firefighting operations are generally expected, but also, in some cases, wind direction may not be the leeward from the breakout point to the NPP so that the forest fire can move away from the NPP, and/or it may rain within hours of the forest fire occurrence. In the sensitivity study, however, the pessimistic case was assumed in order to determine the potential range of the hazard curve by comparing to the reference case where the forest firefighting operation effect is counted.

According to our previous study on the forest fire propagation simulations (Okano and Yamano, 2015b) by using FARSITE, the changeable range of a rate-of-spread (ROS) of the forest fire front propagation is between 0.06 and 3.6 $\mathrm{m} / \mathrm{min}$. The harmonic mean of ROS in the forest fire spread area (i.e. areal averaged harmonic mean of ROS) is between 0.22 and $2.5 \mathrm{~m} / \mathrm{min}$. and the average one is $0.42 \mathrm{~m} / \mathrm{min}$. By using the Weibull distribution for the arrival probability with the Eq. (1), a theoretical estimation is obtained on the arrival probability as a function of a distance from a breakout point to an NPP for each areal averaged harmonic mean of ROS as shown in Fig. 3. For the nominal case (i.e. average harmonic mean of ROS, $0.42 \mathrm{~m} / \mathrm{min}$.), the arrival probability is only 0.1 for the short distance of 0.1 $\mathrm{km}$ from the forest fire breakout point to the NPP. Even for the most severe case (i.e. maximum harmonic mean of ROS, $2.5 \mathrm{~m} / \mathrm{min}$.), the probability is 0.036 for the distance of $1 \mathrm{~km}$. This indicates that most of forest fires in Japan are extinguished before spreading over kilometers, and the conditional probability of forest fire arrival at an NPP is generally very low (e.g. less than a few percent), nevertheless the PRA is indispensable for a quantitative risk evaluation.

Table 1 summarizes the sensitivity study cases mentioned above with the condition parameter sets. Based on our 
previous study, the dotted lines in the left figures of Figs. 4, 5, and 6 show the hazard curves of the reaction intensity of the reference case which show the annual exceedance frequency of $1 \times 10^{-3}$ per year for $800 \mathrm{~kW} / \mathrm{m}^{2}, 1 \times 10^{-4}$ per year for $935 \mathrm{~kW} / \mathrm{m}^{2}$, and about $1 \times 10^{-6}$ per year for $1,050 \mathrm{~kW} / \mathrm{m}^{2}$. The hazard curve of the reaction intensity below $600 \mathrm{~kW} / \mathrm{m}^{2}$ is constant around $5 \times 10^{-3}$ per year and the upper limit of the reaction intensity is around $1,100 \mathrm{~kW} / \mathrm{m}^{2}$; these correspond to the variable range of the reaction intensity. The hazard curves of the fireline intensity show the annual exceedance frequency of $1 \times 10^{-4}$ per year for $107 \mathrm{~kW} / \mathrm{m}$ and about $1 \times 10^{-6}$ per year for $190 \mathrm{~kW} / \mathrm{m}$. Both the hazard curves become around $5 \times 10^{-3}$ per year when the intensity approaches to zero.

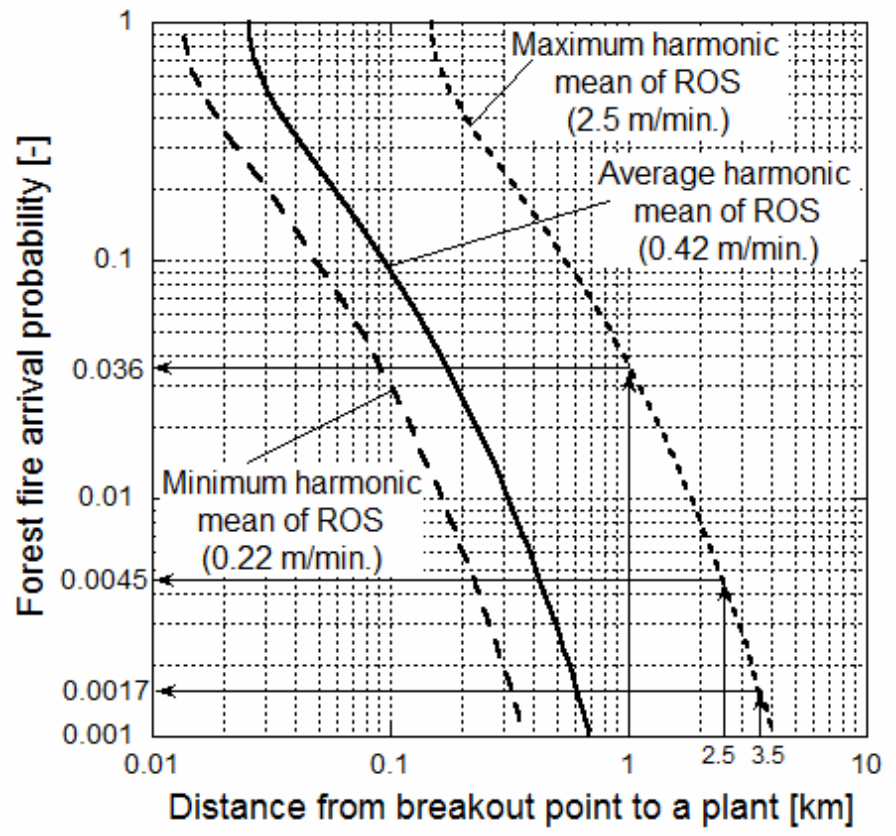

Fig. 3 Forest fire arrival probability is estimated by the Weibull distribution of the forest fire extinguishment probability as a function of a distance from a breakout point to a plant and spatially averaged rate-of-spread (ROS) of forest fire front propagation. For example, the forest fire arrival probability in which the breakout point is $1 \mathrm{~km}$ distant from the plant is estimated to be $0.036(3.6 \%)$ when the forest fire spread speed is the maximum (i.e. $2.5 \mathrm{~m} / \mathrm{min}$.)

Table 1 Probability fluctuation and parameter set of sensitivity study cases, as well as hazard curve sensitivity study results, are shown. Variable parameters are changed from the reference case depending on breakout hourly fluctuation (i.e. daytime biased), breakout point locational fluctuation (i.e. selected one point biased), and with or without forest firefighting operation. Sensitivity study results on reaction intensity and fireline intensity in the sensitivity study cases are compared with those of the reference case.

\begin{tabular}{|c|c|c|c|c|c|c|c|c|c|c|}
\cline { 2 - 9 } \multicolumn{2}{c|}{} & \multicolumn{5}{c|}{ Probability fluctuation and parameter set } & \multicolumn{3}{c|}{ Hazard curve sensitivity } \\
(changes from the reference case)
\end{tabular}




\section{Sensitivity study results and discussions}

Table 1 includes the summary of the hazard curves of the reaction intensity and the fireline intensity evaluated in the sensitivity study with the difference of the frequency or the intensity from those of the reference case.

\subsection{Sensitivity to forest fire breakout time}

The hazard curves considering the fluctuation of breakout time (i.e. high probability of forest fire breakout for daytime peak hour) are shown in solid lines in Fig. 4. In this case, the reaction intensity and the fireline intensity increased $4 \%$ and $14 \%$ from the reference case, respectively. The frequencies of both hazard curves do not change because the hazard curves are "annual" summary (i.e. annual exceedance frequency). The reason for the increase in the intensity is that the breakout probability in daytime peak hour is statistically 24 times higher than nighttime (i.e. 2.8 / $0.11=24$ ), and the intensity in daytime becomes higher than that in nighttime due to the effect of sunshine on the forest. According to the forest fire intensity in relation to sunshine effects (Rothermel et al., 1986), the forest fuel temperature is higher than the ambient air due to sunshine in the daytime:

$$
T_{f f}-T_{a m}=\left(3.56 \times 10^{-4} \times I\right) /(0.015 U+0.0116)
$$

Here, $T$ means temperature (unit in K), the subscriptions $f f$ and $a m$ mean forest fuel and ambient air respectively, $I$ means radiation heat on the forest by sunshine (unit in $\mathrm{W} / \mathrm{m}^{2}$ ), and $U$ means wind speed in the forest (unit in $\mathrm{m} / \mathrm{s}$ ). Due to the forest fuel temperature rise, the RH in the forest decreases (Rothermel et al., 1986):

$$
R H_{f f} / R H_{a m}=\exp \left[-0.0594 \times\left(T_{f f}-T_{a m}\right)\right]
$$

When the parameters are given for the studied area on sunshine radiation heat for north latitude of $36^{\circ}$ (i.e. $I=1.37 \times$ $10^{3}\left(\mathrm{~W} / \mathrm{m}^{2}\right) \times \operatorname{cosine}\left(36^{\circ}\right)$ ) and on wind speed in a forest (e.g. $U=2 \mathrm{~m} / \mathrm{s}$, nearly half of the average PWS of $4.1 \mathrm{~m} / \mathrm{s}$ ), and average $\mathrm{RH}$ for forest fire onset (i.e. $R H_{a m}=49 \%$ ), the representative $\mathrm{RH}$ in the forest is estimated as $R H_{f f}=37 \%$ by using the Eqs. (3) and (4).
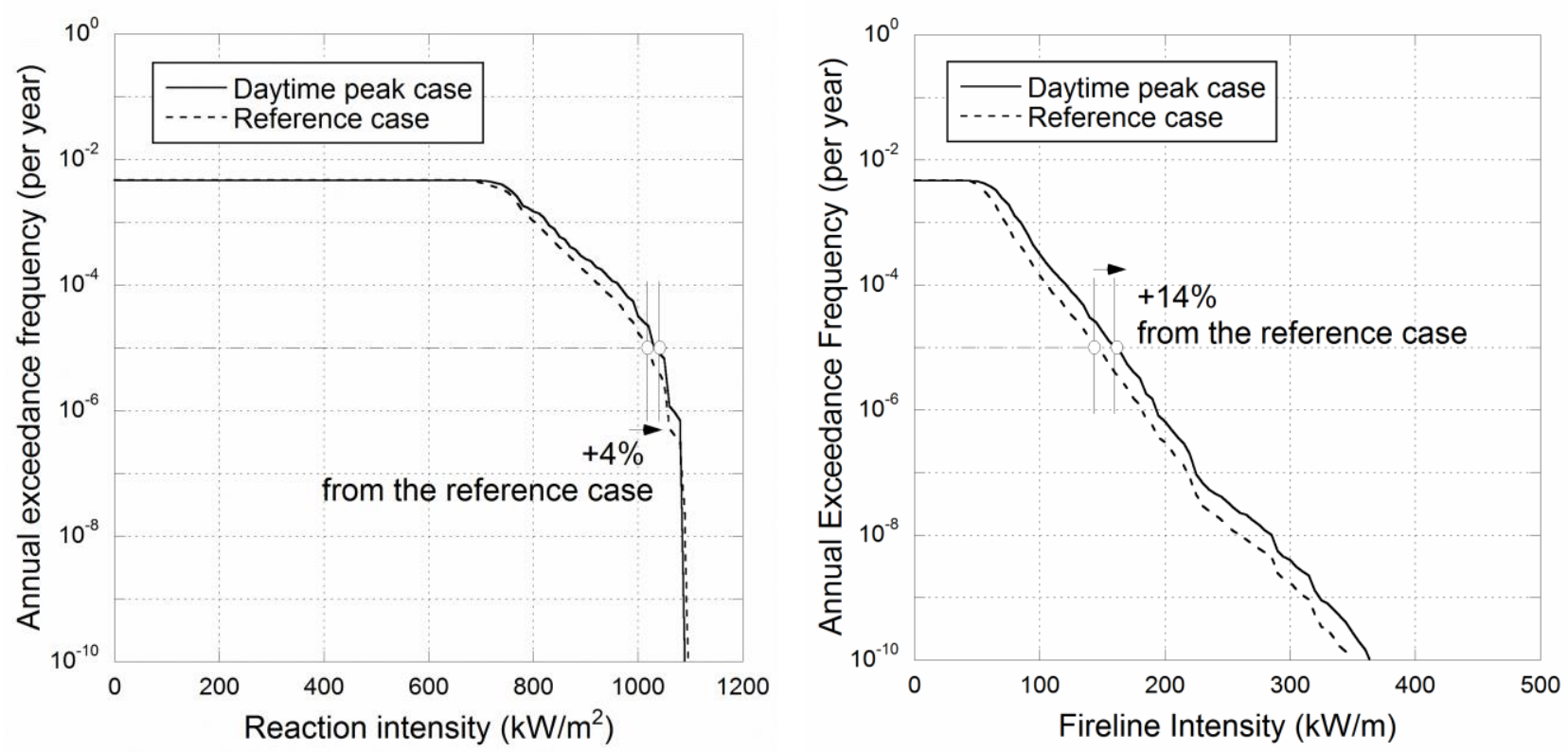

Fig. 4 Hazard curves of reaction intensity (in the left figure) and fireline intensity (in the right figure) for the sensitivity study on the hourly fluctuation of breakout time are depicted in solid lines. Hazard curves of the reference case are depicted in dotted lines. For the annual exceeding frequency of $10^{-5}$ per year, the reaction intensity increases $+4 \%$ and the fireline intensity increases $+14 \%$ on their intensity from those of the reference case. 
Our previous study (Okano and Yamano, 2015b) indicated that the reaction intensity and the fireline intensity decreased due to the RH increases. These changes are simply modeled by using regression formulas:

$$
\begin{aligned}
& I_{r t}=1.0 \times 10^{3}-3.4 \times R H_{a m}, \text { and } \\
& I_{f l}=4.7 \times 10^{2}-2.5 \times R H_{a m} .
\end{aligned}
$$

Here, $I_{r t}$ and $I_{f l}$ mean reaction intensity and fireline intensity, respectively. The daytime $\mathrm{RH}$ in a forest $\left(R H_{f f}=37 \%\right)$ is lower than that in nighttime because the air temperature and $\mathrm{RH}$ in the forest during the nighttime are nearly the same as the ambient air due to the lack of sunshine (i.e. $T_{f f} \cong T_{a m}, R H_{f f} \cong R H_{a m} \cong 49 \%$ ). The low $R H_{f f}$ in the daytime gives the increase of $I_{r t}$ and $I_{f l}$ around $9 \%$ and $15 \%$ respectively according to the Eqs. (5) and (6). These increases are nearly the same as the increases in the hazard curve intensity.

\subsection{Sensitivity to forest fire breakout points}

The solid lines in Fig. 5 show hazard curves with varied probability distribution of the three breakout points. The frequency of the reaction intensity of the three study cases change from $+67 \%$ to $-36 \%$ from the reference case. The frequency of the fireline intensity of the three study cases change from $+58 \%$ to $-41 \%$ from the reference case. These are because the three points have different distances to the NPP and the arrival probability varies with the distances.

A point around $1 \mathrm{~km}$ away from an NPP was set as the nearest potential forest fire breakout point. According to Fig. 3, the arrival probability is 0.036 for $1 \mathrm{~km}$ distance even under the condition with the maximum harmonic mean of ROS. For the other two points with distances of $2.5 \mathrm{~km}$ and $3.5 \mathrm{~km}$ from the NPP, the probability decreases 0.0045 and 0.0017 respectively for the maximum harmonic mean of ROS condition. Therefore, the arrival probability of three breakout points is proportional to the factor of 0.014 (i.e. $(0.036+0.0045+0.0017) / 3=0.014$ ) for the reference case. For the "Point A High" case, the factor becomes 0.023 (i.e. $(0.6 \times 0.036+0.2 \times 0.0045+0.2 \times 0.0017)=0.023)$. The possible change of the frequency is 0.63 (i.e. $(0.023-0.014) / 0.014=0.62=+62 \%)$. For the "Point B High" and "Point C High" cases, the factors become 0.0091 and 0.010 respectively. Then, the possible changes of the frequency are $-35 \%$ and $-27 \%$ respectively. These ranges of fluctuation of $+62 \%,-35 \%$, and $-27 \%$ for the Point $\mathrm{A}, \mathrm{B}, \mathrm{C}$ High cases are almost in the same range of the frequency changes of the reaction intensity and the fireline intensity in the sensitivity study.
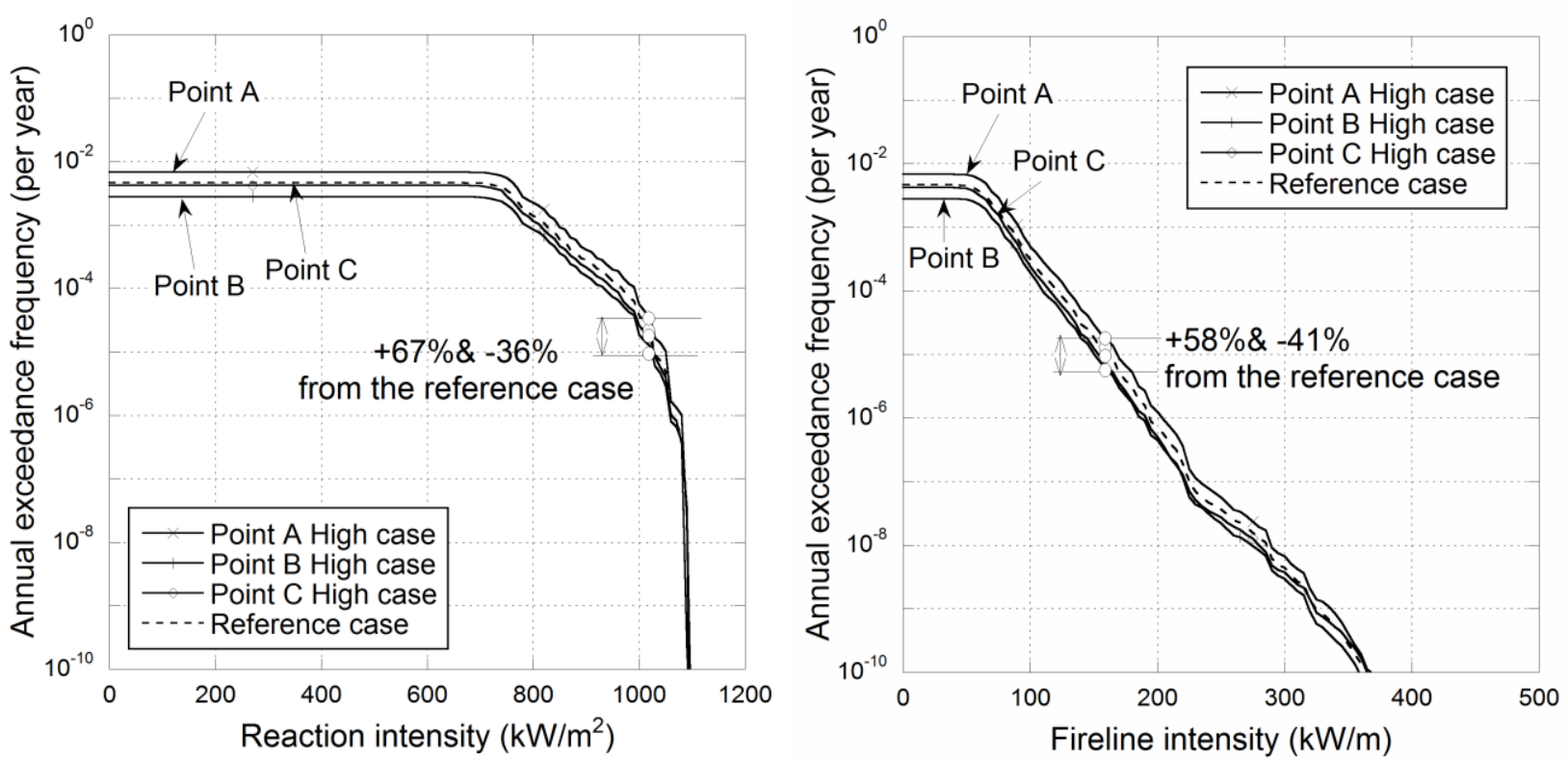

Fig. 5 Hazard curves of reaction intensity (in the left figure) and fireline intensity (in the right figure) for the sensitivity study on the locational fluctuation of breakout points are depicted in solid lines for Point A/B/C High cases. Hazard curves of the reference case are depicted in dotted lines. The reaction intensity changes from $+67 \%$ to $-36 \%$ and the fireline intensity changes from $+58 \%$ to $-41 \%$ on their frequency from those of the reference case at the annual exceeding frequency of $10^{-5}$ per year. 


\subsection{Sensitivity to forest firefighting operation}

The solid lines in Fig. 6 show the hazard curves without considering forest firefighting operation outside an NPP. In other words, the effects of the forest fire extinguishment were excluded. The frequencies of the reaction intensity and the fireline intensity remarkably increased, becoming around 56 times and 38 times higher, respectively, than those of the reference case. In low-intensity with high-frequency situation (e.g. $10 \mathrm{~kW} / \mathrm{m}^{2}$ and $10 \mathrm{~kW} / \mathrm{m}$ ), the frequency increases 77 times higher in both the reaction intensity and the fireline intensity. This is because the probability of the forest fire arrival becomes unity when neglecting effects of the extinguishment by the forest firefighting operations.

According to the probability distribution fluctuation of the forest fire breakout points discussed in Section 4.2, the arrival probability of each breakout point is proportional to the factor of 0.014 . If there is no forest firefighting operation, the probability of forest fire arrival at an NPP increases with the inversed factor: (i.e. 1/0.014 =) 71. This nearly corresponds to the hazard curve frequency increases of 77 times.
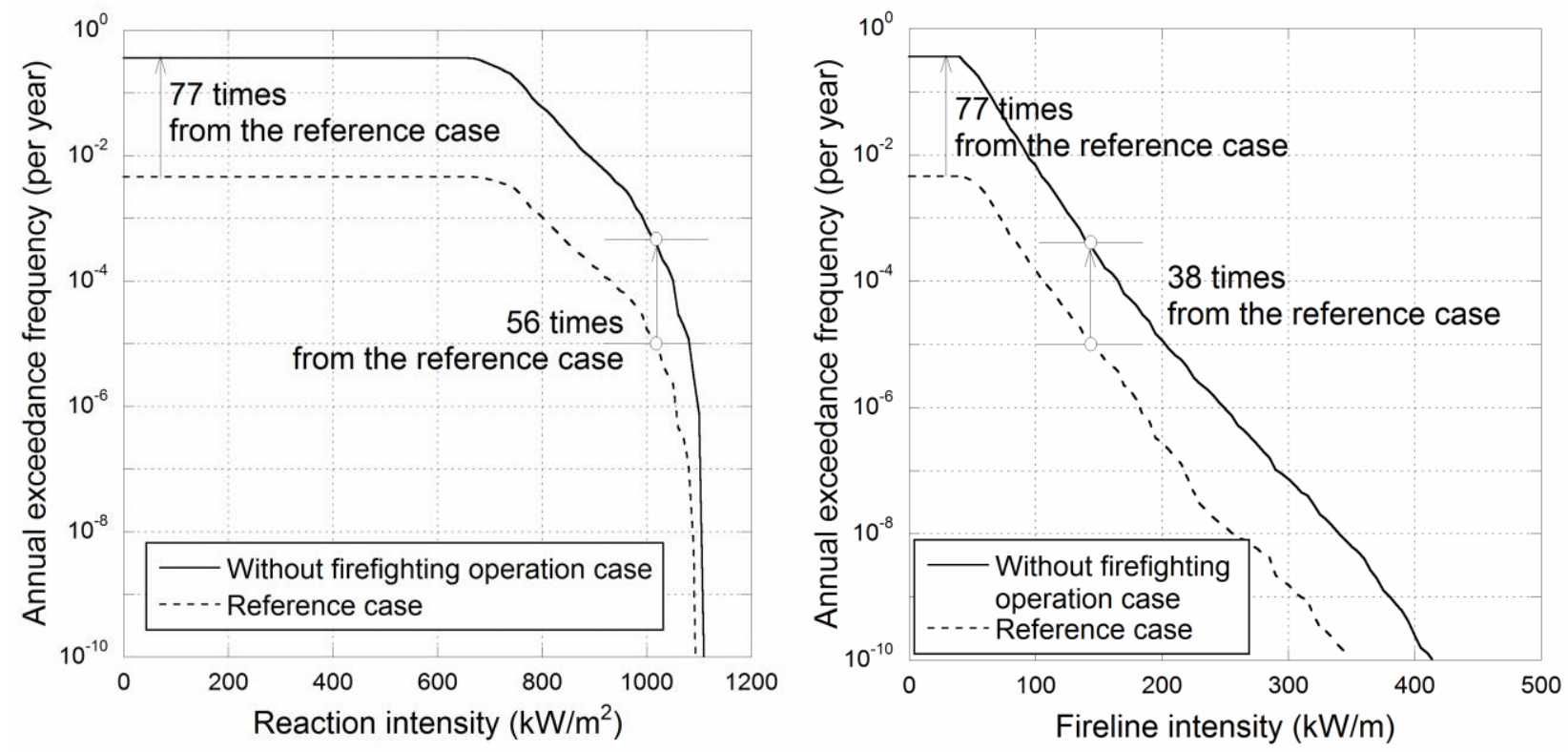

Fig. 6 Hazard curves of reaction intensity (in the left figure) and fireline intensity (in the right figure) for the sensitivity study on the forest fire arrival probability fluctuation due to the effect of forest firefighting operation are depicted in solid lines. Hazard curves of the reference case are depicted in dotted lines. The reaction intensity becomes 56 times and the fireline intensity becomes 38 times on their frequency from those of the reference case at the annual exceeding frequency of $10^{-5}$ per year.

\section{Conclusion}

The sensitivity study on the hazard curves of the reaction intensity and the fireline intensity was performed as a part of a forest fire PRA methodology development. For the sensitivity study, the logic boxes in the logic tree for a forest fire were assessed on their general effects on the frequency and/or intensity of the hazard curve. The study identified variables related to the forest fire breakout time, the forest fire breakout points, and the forest firefighting operation as important parameters. Their fluctuation ranges were evaluated for the sensitivity study based on the statistic investigations. The hazard curves evaluated in this sensitivity study indicated that one of the most significant factors in a forest fire hazard is forest firefighting operations outside an NPP, which are performed during the grace period from the forest fire breakout to the arrival at an NPP. The conditional probability of a forest fire arrival at an NPP from a potential breakout point with kilometer range distance was evaluated to be very low (i.e. less than a few percent). 


\section{Acknowledgments}

The authors wish to thank Mr. T. Inoue of Mitsubishi Research Institute, Inc. on Monte Carlo simulations of hazard curves, and Mr. Y. Goto of the Forestry and Forest Products Research Institute for general advices related to a forest fire and forestry. This study is the result from "Research and Development of Margin Assessment Methodology of Decay Heat Removal Function against External Hazards" entrusted to Japan Atomic Energy Agency by the Ministry of Education, Sports, Science and Technology (MEXT) under Grant System/120802.

\section{References}

Braun W.J., Jones B.L., Lee J.S.W., Woolford D.G., and Wotton B.M., Forest fire risk assessment: an illustrative example from Ontario, Canada, Journal of Probability and Statistics, Vol.2010, Article ID 823018 (2010), http://dx.doi.org/10.1155/2010/823018.

Crisis Measures and Disaster Prevention Division, Forest fire database of Fukui Prefecture (as of December 2013), <http://www.pref.fukui.lg.jp/doc/kikitaisaku/> (in Japanese).

Finney M.A., FARSITE: Fire Area Simulator - Model Development and Evaluation, US Department of Agriculture, RMRS-RP-4 Revised (2004).

Forest Fire Control Association, Practical guide of managements on forest and field fires (the title is translated), Tokyo, Rinya-Kousai-Kai (Japan Forest Foundation) (2007), p.9 (in Japanese).

Forestry Division, Forest Navigation of Fukui Prefecture, available from <http://morinavi.pref.fukui.jp/map.php> (online), (accessed on December, 2013) (in Japanese).

Japan Meteorological Agency, Automated Meteorological Data Acquisition System (AMeDAS), available from <http://www.jma.go.jp/jp/amedas/> (online), (accessed on December, 2013) (in Japanese).

Ministry of Land, Infrastructure, Transport and Tourism (MLIT), National Land Numerical Information, available from <http://nlftp.mlit.go.jp/ksj-e/index.html> (online), (accessed on December, 2013).

Nuclear Regulation Authority (NRA), Guide for assessment on external fire accidents (draft) (the title is translated), NRA, Tokyo, Japan (2013) (in Japanese).

Okano Y. and Yamano H., Hazard curve evaluation method development for a forest fire as an external hazard on nuclear power plants, Journal of Nuclear Science and Technology, Vol.53, No.8 (2015a), pp.1224-1234, DOI: 10.1080/00223131.2015.1102662.

Okano Y. and Yamano H., Forest fire propagation simulations for a risk assessment methodology development for a nuclear power plant, Case Studies in Fire Safety, Vol.4 (2015b), pp.1-10, DOI: http://dx/doi.org/doi:10.1016/j.csfs.2015.05.001.

Okano Y. and Yamano H., Numerical simulations of forest fire propagation and smoke transport as an external hazard assessment methodology development for a nuclear power plant, Mechanical Engineering Journal, Vol.3, No.3 (2016), p.15-00592, DOI: 10.1299/mej.15-00592.

Preisler H.K., Brillinger D.R., Burgan R.E., and Benoit J.W., Probability based models for estimation of wildfire risk, International Journal of Wildland Fire, Vol.13, No.2 (2004), pp. 133-142, http://dx.doi.org/10.1071/WF02061.

Rothermel R. C., Wilson R. A., Jr., Morris G. A., and Sackett S. S., Modeling Moisture Content of Fine Dead Wildland Fuels: Input to the BEHAVE fire prediction system, US Department of Agriculture, INT-359 (1986).

Sano T., Forest fire danger prediction in Hiroshima prefecture and experimental investigating of fire spread danger map, Proceedings of 5th International Symposium on Forest Fire Protection (5th NRIFD) (2005), pp.168-181.

Satoh K., Human causes affecting forest fire danger rating, Proceedings of 5th NRIFD (2005), pp.91-105.

Song W., Wang J., Satoh K., and Weicheng F., Three types of power-law distribution of forest fire in Japan, Ecological Modelling, Vol.196 (2006), pp. 527-532. 